\title{
Contenidos socioculturales en la temática conversacional de un corpus oral bilingüe chino-español de estudiantes de Español Lengua Extranjera
}

\author{
JAVIER PÉREZ RUIZ \\ Wenzao Ursuline University of Languages. Taiwán
}

Recibido: 10 abril 2014 / Aceptado: 12 febrero 2015

ISSN: $1697-7467$

\begin{abstract}
RESUMEN: El objetivo de este artículo es descubrir los contenidos socioculturales de la cultura china-taiwanesa y española presentes en la temática conversacional de un corpus oral bilingüe chino-español de estudiantes taiwaneses de dos niveles lingüísticos de ELE, compuesto por 80 conversaciones, 40 en L1-chino y 40 en L2-español. Los resultados muestran diferencias cuantitativas y cualitativas en los contenidos culturales de las conversaciones con relación al mayor nivel en ELE. Además, el estudio subraya el valor didáctico de la temática sociocultural presente en los corpus orales para la clase de ELE, como ayuda para la adquisición de la competencia comunicativa e intercultural.

Palabras claves: corpus oral, temática conversacional, competencia intercultural, español lengua extranjera.
\end{abstract}

\section{Sociocultural Contents of Conversational Topics in a Spoken Chinese-Spanish Bilingual Corpus of Spanish Foreign Language Learners}

\begin{abstract}
The aim of this paper is to discover the sociocultural references of ChineseTaiwanese and Spanish cultures in an oral Chinese-Spanish bilingual corpus of Taiwanese students, from two different proficiency levels in Spanish foreign language (SFL), comprised from 80 conversations, 40 in Chinese L1 and 40 in Spanish L2. The findings show quantitative and qualitative differences in the cultural topics of conversations related to the proficiency level in SFL. Moreover, the study underlines the didactic value for the SFL classroom of sociocultural conversation topics involved in spoken corpora to increase the acquisition of communicative and intercultural competences.

Keywords: oral corpus, conversational topics, intercultural competence, Spanish foreign language.
\end{abstract}

\section{INTRODUCCIÓN}

La adquisición de la competencia intercultural (CI) conlleva, por la idiosincrasia conceptual del término, un entresijo de factores cognitivos, afectivos, comportamentales, comunicativos, y sobre todo, culturales (Meyer, 1991; Oliveras, 2000). En su complejo desarrollo, se percibe un desigual progreso entre los notables logros alcanzados a nivel teórico (Níkleva, 2012; Rico, 2005) e institucional (Consejo de Europa, 2002; Instituto Cervantes, 2006), y los escasos avances didácticos en la clase de español como lengua extranjera (ELE). Cada 
vez se es más consciente del largo camino que queda por recorrer hasta poder concretar formulaciones pedagógicas precisas de la CI en la clase de L2 (Areizaga, 2001; Barros, 2012; Paricio, 2014). Para suplir estas carencias, deberían ser recogidas nuevas propuestas y enfoques originales que investiguen aquellos puntos que, hasta ahora, han sido escasamente analizados.

Uno de los factores menos estudiados de la CI ha sido cómo fomentar la concienciación de la propia cultura $(\mathrm{C} 1)$ y de la cultura meta $(\mathrm{C} 2)$ en los profesores y estudiantes de L2 (Kramsch, 1991). La búsqueda de nuevas herramientas, que faciliten la mencionada reflexión intercultural, sería una valiosa ayuda docente en el proceso de adquisición de la CI. Ante esta situación, nos proponemos analizar las referencias socioculturales de la $\mathrm{C} 1$ y de la $\mathrm{C} 2$ en un corpus oral bilingüe L1-chino y L2-español de estudiantes taiwaneses de ELE de dos niveles lingüísticos diferentes. A tenor de los resultados que obtengamos, podremos sugerir, con base empírica, la contribución didáctica que los corpus orales $(\mathrm{CO})$ bilingües pueden tener para la clase de ELE. Concretamente, como un instrumento sociolingüístico que puede favorecer la toma de conciencia cultural, al descubrir las señas de identidad sociocultural de los estudiantes.

\section{Marco teórico}

En los estudios sobre las secuencias temáticas de la conversación, los investigadores han centrado sus esfuerzos en explorar el cambio de tema o las secuencias de apertura y cierre en diversas lenguas (Downing, 2005; Fernández, 2003; Stokoe, 2000). Mientras que, en el campo de la interculturalidad, una de las tribunas del debate sociolingüístico se sigue preguntando cómo acercar, de manera real, la interculturalidad a la clase de lenguas extranjeras (Barros, 2012; Byram, 2002; Paricio, 2014; Rico, 2005). Esta tarea integradora de lengua-interacción-interculturalidad viene marcada por complejos desafíos, a saber, la adecuada formación del profesorado y la búsqueda de materiales idóneos para el proceso de adquisición de la CI (Areizaga, 2001; Paricio, 2014). En esta empresa, varios estudios han profundizado sobre los elementos culturales presentes en los manuales y en la búsqueda de nuevos métodos para la adquisición de la CI (Areizaga, 2002; Marín, 2012; Níkleva, 2012; Ruiz, 2004). Desafortunadamente, el análisis de la temática sociocultural en muestras reales de habla, como son los CO bilingües, es una tarea original, que hasta ahora no ha sido debidamente emprendida. Por otra parte, Ortí (2004) indicaba que las conversaciones monoculturales exhibían patrones conversacionales comunicativos diferentes a las conversaciones interculturales.

\subsection{Competencia intercultural y didáctica de la interculturalidad}

De manera resumida, en este apartado, nos ceñiremos a exponer algunas pinceladas generales de la CI y de sus dificultades didácticas. Entendemos por CI aquella competencia más amplia del hablante extranjero que le capacita para comportarse en sus acciones, actitudes y expectativas, de manera adecuada y flexible, en sus encuentros interculturales (Meyer, 1991: 137; Oliveras, 2000). Para Barros (2012), la CI es la habilidad del estudiante de realizar situaciones de comunicación intercultural, por lo que sería una macro competencia 
que incluiría la competencia comunicativa y lingüística. Además, la interculturalidad establece una relación de respeto y de reconocimiento de valores y formas de vida entre personas de diferentes culturas (Níkleva, 2012). En su revisión del tema, Rico (2005) definía la CI como una triple habilidad cognitiva, afectiva y comportamental que permite a personas de diferentes culturas mantener una comunicación efectiva. Sin embargo, para Iglesias (2003) determinados factores pueden influir de manera negativa en la comunicación intercultural, a saber, los estereotipos, los prejuicios, la discriminación y el etnocentrismo.

El Marco común europeo de referencia para las lenguas (MCER) recomendaba la concienciación de la C1 y C2 (Consejo de Europa, 2002). Además, el Instituto Cervantes subrayaba la riqueza de la interculturalidad, que debía desarrollar el sentimiento de identidad cultural de los estudiantes (Instituto Cervantes, 2002). Santamaría (2008) apostaba por la promoción de los aspectos culturales de la $\mathrm{C} 1$ y la $\mathrm{C} 2$ en la clase, creando espacios de interacción en el aula para que los estudiantes puedan desenvolverse en situaciones interculturales.

Sin embargo, una de las principales dificultades de la CI es pasar de su formulación teórico-conceptual a su aplicación práctica en la clase de lenguas extranjeras, es decir, cómo adaptar los currículos y la formación adecuada del profesorado (Barros, 2012). Paricio (2014) declaraba que, en la actualidad, se apreciaba en la formación de los profesores una falta de atención al componente intercultural, con prácticas educativas deficientes.

La perspectiva intercultural en la enseñanza de lenguas aparece a partir de la década de los años 80, ya que los enfoques comunicativos no habían considerado adecuadamente la relación entre lenguaje y cultura (Paricio, 2012). En este nuevo enfoque, se defiende una enseñanza integral de la lengua y la cultura (Barros, 2012; Níkleva, 2012). Pero desgraciadamente, en la actualidad, Barros (2012) comprobó que seguía existiendo una falta de integración entre los contenidos culturales y lingüísticos. Para lograr este objetivo, una de las tendencias actuales proviene de los estudios de Areizaga (2001), quien defendía pasar del enfoque informativo de la cultura, que concebía la cultura como información, al enfoque formativo, en el que la cultura contribuye a la adquisición de la competencia comunicativa intercultural. Para lograr este cambio, se exige una nueva formación del profesorado, que deja de ser un transmisor de repertorios culturales, para ser un mediador en el proceso formativo de la toma de conciencia de la $\mathrm{C} 1$ y de la $\mathrm{C} 2$, favoreciendo la comunicación intercultural (Areizaga, 2001; Byram, 2002; Oliveras, 2000; Paricio, 2014). Además, los profesores deben afrontar nuevos retos, y no limitarse a desmenuzar los elementos culturales presentes en los manuales de textos (Marín, 2012; Níkleva, 2012), abriendo nuevas vías docentes, basadas en muestras reales y actuales que informen sobre el grado de concienciación e identidad cultural de los profesores y estudiantes.

\subsection{Interculturalidad y corpus orales}

Los CO son escasos en el ámbito de ELE debido a la complejidad y esfuerzo que exigen (Pérez, 2014). Sin embargo, son notorias las ventajas que proporcionan en la enseñanza de L2 (Cestero, 2005 y 2012; Seedhouse, 2004); ya que gracias a su input real y variado potencian el enfoque comunicativo de la lengua (McCullough, 2001). Además, para Sinclair (2004) los CO, en un futuro cercano, se convertirán en una herramienta fundamental en todos los ámbitos de la educación, y surgirán nuevas utilidades didácticas.

Por otro lado, varios trabajos permiten vincular el estudio de la CI con los CO. Para García (2004), en la conversación, convergen los temas aprendidos en clase y los conoci- 
mientos socioculturales previos. Paricio (2014: 224) proponía al profesor como aprendiz intercultural, que debe adquirir las mismas destrezas y actitudes que busca en sus estudiantes. Para Byram (2002), debemos ayudar a descubrir la identidad social y las estrategias de interacciones culturales de los alumnos. Rico (2005) propuso una metodología basada en la experiencia, el diálogo y la cooperación entre estudiantes, incorporando muestras auténticas de conversación coloquial; las cuales, muchas veces, habían sido manipuladas, como los diálogos grabados, artificiales en su entonación, pronunciación y contenido. Por último, Unamuno (2003) defendía el papel pedagógico de las grabaciones y transcripciones en la clase.

En definitiva, los $\mathrm{CO}$ pueden convertirse en una valiosa herramienta didáctica para la clase de L2. Al tratarse de muestras reales de habla cotidiana, superan la restricción cultural en la enseñanza que suponía aprender únicamente contenidos informativos, las conocidas cuatro famosas efes de Kramsch (1991: 218), a saber, foods, fairs, folklore and statitical facts. Y, por supuesto, el trabajo con corpus sobrepasa el concepto de miniaturización de la cultura (Scollon, 1995: 382), para poder integrarse en un recurso válido dentro de la nueva concepción formativa de la cultura en la enseñanza de L2 sugerida por varios autores (Areizaga, 2001; Byram, 2002).

Ante estas premisas teóricas, debemos considerar el valor investigador y didáctico que, para la concienciación cultural y, por lo tanto, para la adquisición de la CI, puede tener el análisis de los contenidos socioculturales de la $\mathrm{C} 1$ y de la $\mathrm{C} 2$, presentes en las conversaciones coloquiales entre miembros de una misma cultura.

\section{Metodología}

\subsection{Objetivos del estudio}

El primer objetivo de este estudio es analizar los contenidos socioculturales de la cultura china-taiwanesa, así como de la cultura española y latinoamericana que aparecen en las conversaciones chinas y españolas de un CO bilingüe de estudiantes taiwaneses de ELE. En segundo lugar, se examinará la influencia que el nivel lingüístico adquirido en la L2 puede tener en la producción cuantitativa y cualitativa de las marcas socioculturales halladas. A través de este doble objetivo, esperamos aportar una nueva evidencia empírica sobre la contribución de las conversaciones monoculturales y el uso de los $\mathrm{CO}$ en la adquisición de la CI.

\subsection{Tipo de metodología}

La metodología utilizada recoge las aportaciones del Análisis de la Conversación, el Análisis del Discurso y la Lingüística de Corpus. Se ha seguido una metodología empírica basada en CO, que ha respetado sus fases de elaboración (Hutchby, 2008; Sinclair, 2004; Thomson, 2005). Además, nuestro estudio se define como una investigación descriptiva, no experimental, primaria, básica y transversal (Brown, 1988; Griffin, 2005; Moreno, 1998), que entiende el proceso investigador como un continuo cuantitativo-cualitativo (Bernardo, 2000). Por eso, se ha realizado un estudio de naturaleza cuantitativa y cualitativa que ha examinado las diferencias en el contenido sociocultural presente en un $\mathrm{CO}$ bilingüe con relación al nivel adquirido en ELE. 
Aunque en sentido estricto muchos estudios descriptivos no detallan las variables independientes y dependientes, nosotros optamos por definirlas para una mayor claridad expositiva. De manera que, hemos considerado como variable independiente el nivel lingüístico de ELE, mientras que, las variables dependientes han sido las mediciones cuantitativas del contenido sociocultural en L1 y L2. Por último, en nuestro estudio hemos tipificado los referentes socioculturales como todo enunciado expresado en los turnos y secuencias de habla del CO que identifica de manera específica a una cultura determinada. Por ejemplo, el tema "ir al cine" únicamente se ha contabilizado como marca sociocultural cuando los interlocutores conversaron sobre el cine taiwanés o español, pero no ha sido considerado un distintivo cultural cuando los interlocutores se referían al plan de ir al cine durante el fin de semana.

\subsection{Participantes}

Los resultados de la investigación proceden del análisis de un CO bilingüe chino-español (COBICE) constituido por 80 conversaciones diádicas, 40 en chino y 40 en español como segunda lengua (Pérez, 2014). Un total de 80 informantes taiwaneses estudiantes de ELE han mantenido conversaciones coloquiales de cinco minutos de duración. Cada interlocutor ha participado en dos conversaciones, una en chino mandarín y otra en español con los mismos interlocutores, para favorecer estudios contrastivos. Todos los participantes procedían de la Universidad Wenzao de Lenguas Extranjeras, de Kaohsiung, en Taiwán.

Las variables sociológicas controladas en la muestra han sido la edad, 19-23 años, y el nivel socioeconómico, clase media taiwanesa. Las variables lingüísticas tenidas en cuenta han sido la lengua materna, chino mandarín, y el nivel lingüístico en ELE. Los interlocutores del COBICE eran estudiantes taiwaneses de ELE del nivel B1 y B2, según la certificación del examen DELE y los criterios expresados por el MCER (Consejo de Europa, 2002). El grupo del nivel B1 estaba compuesto por 20 hombres y 20 mujeres, que han estudiado cinco años de español y están a punto de obtener su diplomatura en ELE, mientras que la muestra B2 la componían 35 mujeres y 5 hombres, que han cursado estudios de español durante siete años para conseguir su licenciatura universitaria en ELE.

\subsection{Grabaciones, transcripciones y procesamiento de datos}

Todas las grabaciones fueron realizadas en audio, sin ensayo ni preparación previa; y el grabador no se detuvo durante los cinco minutos que duró la grabación, para así poder registrar las pausas, lapsus y titubeos durante la interacción. A los estudiantes, se les indicó que debían conversar de la manera más natural posible, comenzando su conversación con un saludo y finalizando con una despedida, y que la temática conversacional era libre. También, se les indicó que si durante la conversación no sabían cómo proseguir podían acceder a un listado de temas, que estarían en una página plastificada encima de la mesa, junto al grabador. Solo en el $5 \%$ de la temática total, los interlocutores recurrieron a la lista de temas. La lista contenía una modificación de los temas propuestos por el Proyecto para el Estudio Sociolingüístico del Español de España y América (PRESEEA). También, se siguieron las convenciones del grupo PRESEEA para las transcripciones y etiquetado correspondiente del CO. 


\section{Resultados}

En este apartado, se expondrán los principales resultados cuantitativos y cualitativos de la investigación. En términos generales, hemos hallado un mayor número de referentes culturales en las conversaciones chinas y españolas del grupo B2. En 16 conversaciones chinas del grupo B1, de los 99 temas totales, 37 de ellos tenían un contenido cultural; mientras que, de los 80 temas de las conversaciones en español, se obtuvieron 38 referencias culturales, en 17 conversaciones. En las 20 conversaciones del grupo B2 en L1, la temática total contenía un referente cultural en 78 ocasiones; mientras que, en las 20 conversaciones en L2, aparecieron 64 referencias explícitas culturales. La tabla 1 recoge los principales datos obtenidos.

Tabla 1. Frecuencias y porcentajes de referentes socioculturales en el corpus

\begin{tabular}{|c|c|c|c|c|c|c|c|c|}
\hline \multirow{3}{*}{$\begin{array}{c}\text { Referencias } \\
\text { culturales }\end{array}$} & \multicolumn{4}{|c|}{ Nivel B1 } & \multicolumn{4}{|c|}{ Nivel B2 } \\
\hline & \multicolumn{2}{|c|}{ Chino } & \multicolumn{2}{|c|}{ Español } & \multicolumn{2}{|c|}{ Chino } & \multicolumn{2}{|c|}{ Español } \\
\hline & $\mathrm{Cv}$ & Temas & $\mathrm{Cv}$ & Temas & $\mathrm{Cv}$ & Temas & $\mathbf{C v}$ & Temas \\
\hline Total temas & 20 & 99 & 20 & 80 & 20 & 159 & 20 & 122 \\
\hline Contenido SC & 16 & $37(37 \%)$ & 17 & $38(42 \%)$ & 20 & $78(54 \%)$ & 20 & $\begin{array}{c}64 \\
(52 \%)\end{array}$ \\
\hline $\mathrm{C1}$ & 11 & $22(22 \%)$ & 12 & $22(27 \%)$ & 20 & $60(38 \%)$ & 17 & $33(27 \%)$ \\
\hline $\mathrm{C} 2$ & 5 & $5(5 \%)$ & 5 & $7(9 \%)$ & 10 & $15(10 \%)$ & 11 & $25(20 \%)$ \\
\hline $\mathrm{C} 3$ & 6 & $8(8 \%)$ & 6 & $8(10 \%)$ & 2 & $2(1 \%)$ & 3 & $4(3 \%)$ \\
\hline C4-5 & 2 & $2(2 \%)$ & 1 & $1(1 \%)$ & 1 & $1(0.5 \%)$ & 2 & $2(1,5 \%)$ \\
\hline
\end{tabular}

Contenido SC: contenido sociocultural. $\mathrm{C} 1$ : cultura china y taiwanesa.

C2: cultura española y latinoamericana. C3: cultura anglosajona. C4-5: cultura japonesa y coreana.

Cv: número de conversaciones

Al desglosar los contenidos socioculturales por culturas, observamos que en ambos grupos y lenguas han predominado los referentes culturales hacia su propia $\mathrm{C} 1$. Concretamente, tanto en las conversaciones en L1 como en L2 del grupo B1, se objetivaron 22 referencias a la cultura china y taiwanesa. En las conversaciones en L1 del grupo B2, se hallaron 60 marcas socioculturales; y en las conversaciones en L2 se contabilizaron 33.

En segundo lugar, en el grupo B1, se evidenció una mayor presencia de marcas culturales del mundo anglosajón que del mundo hispánico. Por el contrario, en los estudiantes del grupo B2, fue evidente el predominio de marcas socioculturales explícitas hacia el mundo español y latinoamericano frente a los aislados referentes al mundo anglosajón. Por último, y a distancia, en ambos grupos, las culturas coreana y japonesa completaron el mosaico cultural presente en nuestro COBICE.

Además de la estadística descriptiva, hemos querido realizar una comparación estadística inferencial para definir, de manera más precisa, las diferencias halladas. En la tabla 2, mostramos los principales resultados hallados. 
Tabla 2. Resultados estadísticos de las referencias socioculturales en el corpus

\begin{tabular}{|c|c|c|c|c|}
\hline $\begin{array}{c}\text { Conversaciones } \\
\text { chino }\end{array}$ & Grupo & Media & $\begin{array}{l}\text { Desviación } \\
\text { Típica }\end{array}$ & T de Student \\
\hline \multirow{2}{*}{ Referencias culturales } & B1 & 1,85 & 1,31 & \multirow{2}{*}{$\mathbf{0 , 0 0 * *}$} \\
\hline & B2 & 3,75 & 0,97 & \\
\hline \multirow{2}{*}{$\mathrm{C} 1$} & B1 & 1,10 & 1,12 & \multirow{2}{*}{$0,00^{* *}$} \\
\hline & B2 & 3,00 & 0,32 & \\
\hline \multirow{2}{*}{$\mathrm{C} 2$} & B1 & 0,25 & 0,44 & \multirow{2}{*}{$0,03 *$} \\
\hline & $\mathrm{B} 2$ & 0,75 & 0,91 & \\
\hline \multirow{2}{*}{$\mathrm{C} 3$} & B1 & 0,40 & 0,68 & \multirow{2}{*}{0,16} \\
\hline & B2 & 0,15 & 0,37 & \\
\hline $\begin{array}{c}\text { Conversaciones } \\
\text { español }\end{array}$ & Grupo & Media & $\begin{array}{l}\text { Desviación } \\
\text { Típica }\end{array}$ & T de Student \\
\hline \multirow{2}{*}{ Referencias culturales } & B1 & 1,90 & 1,25 & \multirow{2}{*}{$0,00 * *$} \\
\hline & $\mathrm{B} 2$ & 3,20 & 1,40 & \\
\hline \multirow{2}{*}{$\mathrm{C} 1$} & B1 & 1,10 & 0,79 & \multirow{2}{*}{0,08} \\
\hline & B2 & 1,65 & 1,09 & \\
\hline \multirow{2}{*}{$\mathrm{C} 2$} & B1 & 0,35 & 0,67 & \multirow{2}{*}{$0,00 * *$} \\
\hline & B2 & 1,25 & 0,97 & \\
\hline \multirow{2}{*}{$\mathrm{C} 3$} & B1 & 0,35 & 0,75 & \multirow{2}{*}{0,6} \\
\hline & B2 & 0,25 & 0,55 & \\
\hline \multirow{2}{*}{ Ref. cult. repetidas } & B1 & 0,60 & 0,50 & \multirow{2}{*}{$0,04 *$} \\
\hline & B2 & 1,20 & 1,11 & \\
\hline Correlación cultural & Lengua & Correl & ción Pearson & $\begin{array}{l}\text { Significación } \\
\text { Pearson }\end{array}$ \\
\hline \multirow{2}{*}{ Ref. culturales B1 } & L1 & \multirow{2}{*}{\multicolumn{2}{|c|}{0,34}} & \multirow{2}{*}{0,14} \\
\hline & L2 & & & \\
\hline \multirow{2}{*}{ Ref. culturales B2 } & L1 & \multirow{2}{*}{\multicolumn{2}{|c|}{0,55}} & \multirow{2}{*}{$0,048 *$} \\
\hline & $\mathrm{L} 2$ & & & \\
\hline \multicolumn{5}{|c|}{$\begin{array}{l}\text { Ref. cult:: referencias culturales. C1: cultura china y taiwanesa. } \\
\text { C2: cultura española y latinoamericana. C3: cultura anglosajona. } \\
\mathrm{n}=40 \mathrm{p}<0,01 \text {. }\end{array}$} \\
\hline
\end{tabular}


Hemos obtenido diferencias estadísticamente significativas, con un nivel de significación estadística del 0,01 , en el número de referencias culturales generales y de la cultura china en las conversaciones en L1 entabladas en ambos grupos $(p=0,00, p<0,01)$. Mientras que, las referencias a la cultura española y latinoamericana en las conversaciones en chino mostraron diferencias significativas entre los dos grupos para un índice de significación del $0,05(\mathrm{p}=0,03, \mathrm{p}<0,05)$, por lo que se exige una muestra mayor para poder confirmar tales diferencias. En las conversaciones en L2 mantenidas por los grupos B1 y B2, se encontraron diferencias estadísticamente significativas en las marcas socioculturales generales y en las referencias a la $\mathrm{C} 2(\mathrm{p}=0,00, \mathrm{p}<0,01)$.

También, hemos investigado el número de referentes culturales repetidos en las conversaciones de ambas lenguas en cada grupo. Nuestros resultados hallaron que en 14 conversaciones del grupo B1 se repitieron 12 referentes culturales; y en el grupo B2, la misma temática sociocultural estuvo presente en 16 conversaciones y en 23 ocasiones. La T de Student mostró una significación estadística al 5\% $(\mathrm{p}=0,04, \mathrm{p}<0,05)$. En el grupo $\mathrm{B} 1$, los viajes por Taiwán fueron el distintivo cultural más repetido; mientras que, en el grupo B2, las discusiones sobre el servicio militar, el trabajo en Latinoamérica y los viajes a España fueron los temas culturales en los que más reincidieron.

Nuestro estudio estadístico se completó analizando la correlación estadística existente dentro de cada grupo. Puesto que los mismos interlocutores conversaron en ambas lenguas, quisimos conocer la presencia de un probable patrón sociocultural. Los resultados de la Correlación de Pearson llevada a cabo con relación a las estrategias socioculturales generales presentes en el COBICE indican solamente una ligera correlación positiva y significativa al $5 \%$ en el grupo B2 $(r=+0,55, \mathrm{p}<0,05)$.

Intencionadamente, finalizaremos la presentación de los resultados cuantitativos con el desglose de la temática sociocultural china-taiwanesa y española-latinoamericana, así como de su frecuencia, ya que su contenido cuantitativo-cualitativo nos permitirá exponer, de manera más evidente, los principales resultados cualitativos hallados en nuestro CO. Para simplificar su exposición, en la tabla 3, se presenta la temática conversacional distribuida por grupos, pero no por la lengua en la que se conversó.

Tabla 3. Frecuencia de la temática sociocultural de la C1 y de la C2

\begin{tabular}{|l|l|}
\hline \multicolumn{2}{|c|}{ Grupo B1: temática sociocultural china-taiwanesa (44 referentes) } \\
\hline 1. Turismo por Taiwán (4). & 10. Estudio de la cultura china (2). \\
2. Música taiwanesa (3). & 11. Historia de Taiwán (2). \\
3. Comida taiwanesa (3). & 12. Museos y exposiciones taiwanesas (2). \\
4. Cine chino (3). & 13. Familia separada trabajo en China (2). \\
5. Mercado nocturno (3). & 14. Cultura del tentempié nocturno (2). \\
6. Deporte como cultura (3). & 15. Cultura de comer en la calle (2). \\
7. Año Nuevo Chino (3). & 16. Karaoke (2). \\
8. Servicio militar obligatorio (3). & 17. Disminución de la natalidad (1). \\
9. Vida diaria de un estudiante taiwanés (3). & 18. Deporte chino: taichí (1). \\
\hline
\end{tabular}




\begin{tabular}{|c|c|}
\hline \multicolumn{2}{|c|}{ Grupo B2: temática sociocultural china-taiwanesa (93 referentes) } \\
\hline $\begin{array}{l}\text { 1. Educación: diferencias aprendizaje de una } \\
\text { lengua: memorización en el aprendizaje (5). } \\
\text { 2. Relación de respeto profesor y alumnos (5). } \\
\text { 3. Comida china: soja, fideos de Sichuan, } \\
\text { cultura del té, comida vegetariana (5). } \\
\text { 4. Mercado nocturno y tentempié nocturno (4). } \\
\text { 5. Concepto de salud china: papel del agua, } \\
\text { descanso, comida, protección solar (4). } \\
\text { 6. Medicina china y sistema médico chino (4). } \\
\text { 7. Cena de graduación y agradecimiento a los } \\
\text { profesores (4). } \\
\text { 8. Turismo taiwanés y duración vacaciones (4). } \\
\text { 9. Peculiaridades en las fiestas chinas (4). } \\
\text { 10. Música taiwanesa y china (4). } \\
\text { 11. Cine taiwanés y chino (4). } \\
\text { 12. Historia de Taiwán y sus ciudades (4). } \\
\text { 13. Diferencias económicas y laborales norte y } \\
\text { sur de Taiwán (4). }\end{array}$ & $\begin{array}{l}\text { 14. Academias privadas taiwanesas (3). } \\
\text { 15. Servicio militar obligatorio (3). } \\
\text { 16. Cultura del deporte: día del deporte (3). } \\
\text { 17. Ejercicios chinos: yoga y taichí (3). } \\
\text { 18. Orgullo por el desarrollo de Taiwán (3). } \\
\text { 19. Promoción internacional de Taiwán (3). } \\
\text { 20. Diferencias con China (3). } \\
\text { 21. Militares: disciplina en la universidad (3). } \\
\text { 22. Cultura de la moto en Taiwán (3). } \\
\text { 23. Cultura del karaoke (2). } \\
\text { 24. Concepto de belleza china: piel blanca } \\
\text { (2). } \\
\text { 25. Cultura ecológica: hombre y naturaleza } \\
\text { (2). } \\
\text { 26. Templo y dioses chinos (2). } \\
\text { 27. Representación de títeres chinos (1). } \\
\text { 28. Examen de selectividad chino (1). } \\
\text { 29. Machismo en Taiwán (1). }\end{array}$ \\
\hline \multicolumn{2}{|c|}{ Grupo B1: temática sociocultural española e hispanoamericana (12 referentes) } \\
\hline $\begin{array}{l}\text { 1. Viaje a España (2). } \\
\text { 2. Música española (2). } \\
\text { 3. Cine español (2). } \\
\text { 4. Estudiar español (2). }\end{array}$ & $\begin{array}{ll}\text { 5. } & \text { El examen DELE (2). } \\
\text { 6. } & \text { Cultura española (1) } \\
\text { 7. } & \text { Gastronomía española (1). }\end{array}$ \\
\hline \multicolumn{2}{|c|}{ Grupo B2: temática sociocultural española e hispanoamericana (40 referentes) } \\
\hline $\begin{array}{l}\text { 1. } \text { Comercio Hispanoamérica (4). } \\
\text { 2. Estudiar en universidades españolas (3). } \\
\text { 3. Profesores y clases de español (3). } \\
\text { 4. Viaje a España (3). } \\
\text { 5. Música española: cantantes actuales (3). } \\
\text { 6. Comercio y economía en España (2). } \\
\text { 7. Cultura Inca (2). } \\
\text { 8. Nicaragua (2). } \\
\text { 9. Perú (2). } \\
\text { 10. } \\
\text { Relaciones Latinoamérica (2). }\end{array}$ & $\begin{array}{l}\text { 11. Flamenco (2). } \\
\text { 12. Salsa (2). } \\
\text { 13. Monumentos españoles (2). } \\
\text { 14. Comida española (2). } \\
\text { 15. Arte español (1). } \\
\text { 16. Literatura española (1). } \\
\text { 17. Comida de Latinoamérica (1). } \\
\text { 18. Cultura española en general (1). } \\
\text { 19. Futbol (1). } \\
\text { 20. Política en España (1). }\end{array}$ \\
\hline
\end{tabular}

Además de las ya mencionadas diferencias cuantitativas, hemos querido realizar un análisis cualitativo para poder apreciar, de manera más diáfana, las manifiestas diferencias observadas en los contenidos socioculturales de la $\mathrm{C} 1$ y de la $\mathrm{C} 2$ entre los dos grupos estudiados. En primer lugar, en las conversaciones del grupo B1, los referentes culturales contenidos siempre han sido escasos y marginales, con marcas culturales presentes en un único par adyacente, constituido por dos turnos muy breves, sin nuevos remas, y por lo tanto, los interlocutores no han construido secuencias temáticas culturales. Sin embargo, 
la interacción oral del grupo B2 se ha caracterizado por una mayor riqueza cualitativa en la construcción del turno y en la progresión de la temática cultural. Los pares adyacentes han ido encadenando secuencias temáticas culturales, principalmente lineales simples, en las que un tema recibe por parte del oyente una nueva información o rema cultural que, posteriormente, se tematizaba. Fundamentalmente, la progresión temática cultural ha seguido estrategias de tema continuo, en el que al tema principal se le añadían nuevas informaciones.

En segundo lugar, observamos una mayor diversidad y riqueza de la temática cultural de la $\mathrm{C} 1$ y $\mathrm{C} 2$ presentes en el grupo B2. En el grupo B1, tanto en L1 como en L2, las señas socioculturales siempre han sido escasas en extensión, normalmente aparecieron en una única frase; y además, fueron superficiales en su contenido, ya que se limitaban a expresar gustos o deseos sencillos. En este grupo, los temas culturales más repetidos fueron los viajes, la música, la comida y el cine. Por el contrario, en el grupo B2, ha sorprendido la riqueza de los referentes culturales contenida. Estos interlocutores se han prodigado en abordar la temática sociocultural con mayor profundidad, extensión, riqueza de matices y sobre todo, un mayor espíritu crítico y una mayor capacidad para debatir, en ambas lenguas, temas de contenido político y educativo. Han predominado los temas culturales relacionados con las diferencias de la enseñanza entre oriente y occidente. También, ha destacado la presencia de aspectos culinarios específicos, marcas distintivas propias de la salud y la cultura del deporte heredadas de Confucio, la defensa del patrimonio turístico y cultural de Taiwán, así como las manifestaciones artísticas autóctonas.

Las marcas culturales de la cultura española y latinoamericana han evidenciado una notable diferencia cuantitativa y cualitativa entre los grupos B1 y B2. En el grupo B1, se observó una escasez de referentes culturales hacia la cultura española, y una llamativa ausencia de marcas sociológicas de la cultura latinoamericana. En las conversaciones chinas, solo se hallaron 5 referencias en 5 conversaciones. De las cuales, en dos ocasiones, se percibió una actitud positiva hacia la $\mathrm{C} 2$, mientras que en tres ocasiones, se percibió un sentimiento negativo o de rechazo hacia lo español. En las conversaciones en español, también se observaron 7 escasos referentes culturales hacia la cultura española en 5 conversaciones. Uno de ellos mostró una percepción negativa expresada en el deseo de abandonar los estudios de español porque el inglés es la lengua del mundo.

Por su parte, los estudiantes del grupo B2 presentaron un mayor interés y motivación hacia la cultura española y latinoamericana. Además, siempre hubo una percepción sociolingüística positiva hacia el mundo cultural español y latinoamericano. Las principales marcas socioculturales versaron sobre el comercio con Latinoamérica, proseguir estudios relacionados con el mundo del español, los profesores y las clases de español, así como otros referentes turísticos y trazas socioculturales de Latinoamérica, además del baile flamenco y la salsa; y por último, la presencia de ciertas inquietudes culturales muy favorables hacia el arte y la literatura española.

Por último, el análisis de las marcas sociológicas de otras culturas mostró que, en términos cuantitativos y cualitativos, las referencias socioculturales hacia la lengua y cultura anglosajonas superaron a las de la lengua y cultura españolas en el grupo B1. En este grupo se observó una percepción sociolingüística más favorable hacia la cultura inglesa. Aunque, en el grupo B2, los trazos culturales y afectivos del mundo español dominaron frente a los de la cultura anglosajona, también destacó una marcada presencia del prestigio social hacia la lengua y la cultura inglesa en el mencionado grupo. 


\section{Discusión}

En este apartado, centraremos nuestra discusión en la contribución que, para la adquisición de la CI en ELE, pueden tener las referencias socioculturales y las diferencias halladas en el CO analizado. Además, analizaremos el significado de las conversaciones monoculturales en el desarrollo de la interculturalidad. Por último, a la luz de los resultados obtenidos, examinaremos el valor didáctico de los corpus bilingües en la clase de ELE.

Nuestro corpus confirma que las palabras y enunciados gramaticales, más allá de su significado semántico expresan en sí mismos una cultura propia (Eggins, 2003; Gallardo, 1996; Marín, 2012). Nuestras conversaciones han proporcionado muestras actuales y reales de habla coloquial que, además de análisis lingüísticos y comunicativos, permiten descubrir los tres tipos de cultura que mencionaban Miquel y Sans (1992), a saber, la cultura con mayúsculas, la cultura a secas, y la cultura con $k$. Pero, sobre todo, los contenidos socioculturales hallados en el COBICE han confirmado lo publicado por Paricio (2014), quien indicaba la prevalencia actual del concepto de cultura antropológico o cultura con c minúscula, para referirse a la vida diaria, tradiciones y costumbres de una determinada comunidad. Estos tres elementos han estado presentes en las conversaciones de nuestro $\mathrm{CO}$, que ha ofrecido un mosaico pluricultural de cinco culturas diferentes.

Desgraciadamente, no basta con enumerar las referencias socioculturales de un CO, sino que se debe profundizar en la dimensión cultural específica de cada una de ellas, por ejemplo, el diferente sentido del concepto chino-taiwanés de la salud, el deporte, la ecología y la religión contenido en el CO. Estas ideas encierran una identidad cultural particular, heredada y asumida, que poseen un significado diferente a nuestra cultura occidental, y que debería conocer el profesor de L2 para favorecer un adecuado desarrollo de la CI en la clase de ELE. Por tanto, tal como otros autores han defendido, nuestros hallazgos confirman la necesidad de crear en la clase de L2 espacios de interacción intercultural y reflexión compartida en el que afloren las creencias culturales de nuestros estudiantes (Valls, 2011) y en el que se puedan reconocer y respetar los valores culturales (Níkleva, 2012).

Las diferencias cuantitativas y cualitativas observadas con relación al nivel lingüístico en L2 permiten realizar tres interesantes reflexiones. En primer lugar, nuestros resultados indican que la futura adquisición de la CI debe implicar la participación de la triple capacidad cognitiva, afectiva y comportamental, ya expresada por Rico (2005). En los estudiantes del grupo B2, el nivel adquirido de ELE y los años de contacto con la lengua han determinado actitudes más positivas hacia el deseo de ampliar sus conocimientos, así como un mayor acercamiento emotivo y profesional hacia la cultura hispanoamericana. Esta aproximación cognitiva, psicológica y social observada debería facilitar una comunicación más efectiva con personas de la C2. En segundo lugar, la mayor actitud positiva hacia la $\mathrm{C} 1$ y C2, observada en los estudiantes del grupo B2, confirma los resultados de Pérez (2011), quien documentó una percepción sociolingüística más favorable hacia la $\mathrm{C} 1$ y la $\mathrm{C} 2$ en los estudiantes taiwaneses de inglés y español de mayor nivel en su L2. Por último, la correlación observada en las conversaciones chinas y españolas del grupo B2 también está en consonancia con estudios previos llevados a cabo en otras muestras de habla (Pérez, 2012 y 2014 en prensa). En los mencionados estudios, Pérez observó que el mayor nivel lingüístico de los estudiantes les permitía desarrollar estrategias discursivas más dirigidas a la interacción, y por tanto, se 
apreciaba una mayor cercanía en los patrones comunicativos de su L1 y L2. En nuestro estudio actual, el mayor número de referentes socioculturales repetidos en el grupo B2 indica una aproximación cultural en las conversaciones chinas y españolas.

Por todo lo anteriormente expuesto, nuestro estudio plantea dos sugerencias pedagógicas fundamentales. En primer lugar, recomienda promover enfoques didácticos culturales desde estadios más precoces en el desarrollo de la CI, tal y como otros autores han sugerido (Byram 2002, Sanhueza, 2012). Las notorias carencias cuantitativas y cualitativas halladas en la temática sociocultural de los estudiantes del grupo B1 sugieren que, quizás, deberíamos adelantar el acercamiento cultural en nuestros planes curriculares y proponer enfoques didácticos culturales formativos desde las primeras etapas del aprendizaje de ELE.

En segundo lugar, el contenido lingüístico, emocional y sociocultural presente en nuestro CO bilingüe monocultural permite plantear dos recomendaciones. Por un lado, debemos redescubrir el valor de las conversaciones monoculturales, las cuales pueden construir patrones de comunicación diferentes a las conversaciones interculturales (Ortí, 2004). Si comprendemos y explotamos didácticamente la información contenida en los CO bilingües monoculturales podremos establecer una base, a partir de la cual, se podrán diseñar y desarrollar las futuras conversaciones interculturales. Por otro lado, nuestros resultados indican que los $\mathrm{CO}$ pueden convertirse en una herramienta sociolingüística para desarrollar la concienciación cultural, requisito esencial para la adquisición de la $\mathrm{CI}$. Confiamos en que los $\mathrm{CO}$, como fuente de material sociocultural auténtico y actual, permitan a los profesores y a los estudiantes fomentar el mutuo conocimiento cultural a través de la creación de espacios de interacción oral, basados en el reconocimiento y respeto hacia la concienciación de la especificidad y de la diversidad cultural. De este modo, la utilización de los CO podrá contribuir a la adquisición de la CI.

\section{Conclusiones}

Las principales conclusiones del presente estudio muestran que, el análisis de un $\mathrm{CO}$ bilingüe chino-español, compuesto por ochenta conversaciones diádicas entre estudiantes taiwaneses de nivel B1 y B2 en ELE, ha mostrado diferencias significativas cuantitativas y cualitativas en los referentes socioculturales de la cultura china-taiwanesa y de la cultura española-latinoamericana con relación al nivel lingüístico de los interlocutores.

En líneas generales, las conversaciones del grupo B2 han presentado un mayor número de marcas socioculturales tanto en L1-chino como en L2-español; además, sus señas culturales ofrecieron una mayor variedad, extensión y riqueza en la temática sociocultural. Por último, debe ser subrayado el hecho de que, a mayor nivel lingüístico en la L2 se ha comprobado una percepción sociolingüística más positiva, no solamente hacia la lengua y cultura meta, sino también hacia la propia L1 y C1. Estos hallazgos pueden permitir a los profesores y estudiantes tomar conciencia de ambas culturas y sentar las bases para una comunicación intercultural. De manera que, nuestros resultados proporcionan una nueva evidencia empírica sobre la aportación que los corpus orales bilingües compuestos por conversaciones monoculturales pueden tener en el proceso de la adquisición de la CI. 


\section{Agradecimientos}

Esta investigación ha sido financiada por el Ministerio de Ciencia y Tecnología de Taiwán, República de China. Proyecto de investigación anual: NSC 100-2410-H-160-007.

\section{ReFERENCiAs bibliográficas}

Areizaga, E. (2001). "Cultura para la formación de la competencia comunicativa intercultural: el enfoque formativo", en Revista de Psicodidáctica, 11-12: 157-170.

Areizaga, E. (2002). "El componente cultural en la enseñanza de lenguas: elementos para el análisis y la evaluación del material didáctico", en Cultura y educación 14, 2: 161-175.

Barros García, B. y Kharnásova G.M. (2012). "La interculturalidad como macrocompetencia en la enseñanza de lenguas extranjeras: revisión bibliográfica y conceptual", en Porta Linguarum, 18: 97-114.

Bernardo Carrasco, J. y Calderero Hernández, J. F. (2000). Aprendo a investigar en educación. Madrid: Rialp.

Block, D. y Cameron, D. (2002). Globalization and Language Teaching. New York: Routledge.

Brown, J. D. (1988). Understanding Research in Second Language Learning. Cambridge: C.V.P.

Byram, M., Gribkova, B. y Starkey, H. (2002). Développer la dimension interculturelle del'enseignement des langues. Une introduction à l'usage des enseignants. Strasbourg: Conseil de l'Europe.

Cestero Mancera, A. M. (2005). La conversación y la enseñanza de lenguas extranjeras. Madrid: Arco/Libros.

Cestero Mancera, A.M. (2012). "La enseñanza de la conversación en ELE: Estado de la cuestión y perspectivas de futuro", en Revista Internacional de Lenguas Extranjeras, 1: 31-62.

Consejo de Europa, (2002). Marco común europeo de referencia para las lenguas: aprendizaje, enseñanza, evaluación. Madrid: Anaya.

Downing, A. (2005). "Achieving coherence: topicality, conceptualizations and action sequences in negotiating conflicting goals", en Revista Canaria de Estudios Ingleses, 49: 13-28.

Eggins, S. y Martin J. R. (2003). "El contexto como género: una perspectiva lingüística funcional", en Revista Signos, 36, 54: 185-205.

Fernández Agoero, M. (2003). "Analysis of topicality in classroom discourse: topic switch and topic drift in conversations in EFL contexts", en Estudios Ingleses de la Universidad Complutense, 11: 73-89.

Gallardo Paúls, B. (1996). Análisis conversacional y pragmática del receptor. Valencia: Episteme.

García Sánchez, I. (2004). "Manu chao, análisis sociolingüístico de su cancionero", en Tonos, Revista Electrónica de Estudios Filológicos, 8: 1-41.

Griffin, K. (2005). Lingüística aplicada a la enseñanza del español como 2/L. Madrid: Arco/Libros.

Hjarvard, S. (2004). "The Globalization of Language. How the Media Contribute to the Spread of English and the Emergence of Medialects", en Nordicom Review, 75-97.

Hutchby, I. y Wooffitt, R. (2008). Conversation analysis. Cambridge: Polity Press.

Iglesias Casal, I. (2003). "Construyendo la competencia intercultural: sobre creencias, conocimientos y destrezas", en Carabela, 54: 5-29.

Instituto Cervantes (2006). Plan curricular para la enseñanza del español como lengua extranjera. Madrid: Biblioteca Nueva. 
Kramsch, C. (1991). "Culture in language learning: A view from the States", en K. De Bot, R. B. Ginsberg y C. Kramsch (eds.): Foreign language research in cross-cultural perspective. Amsterdam: John Benjamin, 217-240.

Kubota, R. (2002). "The impact of Globalization on language teaching in Japan", en D. Block. y D. Cameron (eds): Globalization and language teaching. New York: Routledge. 13-28.

Marín Escudero, P. (2012). "Lectura sociocrítica de manuales de ELE. Suplementos MarcoELE, 14: $1-45$.

McCullough, J. L. (2001). "Los usos de los córpora de textos en la enseñanza de lenguas", en T. Parera (ed.): Nuevas tecnologías para el autoaprendizaje y la didáctica de lenguas. Lleida: Milenio, 125-140.

Meyer, M. (1991). "Developing transcultural competence: case studies of advanced foreign language learners", en D. Buttjes y M. Byram (eds.): Mediating Languages and Cultures. Clevedon: Multilingual Matters, 136-158.

Miquel, L. y Sans N. (1992). "El componente cultural: un ingrediente más en las clases de lengua", en Cable, 9: 15-21.

Moreno Fernández, F. (1998). Principios de sociolingüística y sociología del lenguaje. Barcelona: Ariel.

Níkleva, D. G. (2012). "La competencia intercultural y el tratamiento de contenidos culturales en manuales de español como lengua extranjera", en RESLA, 25: 165-187.

Paricio Tato, M.S. (2014). "Competencia intercultural en la enseñanza de lenguas extranjeras", en Porta Linguarum, 21: 215-226.

Oliveras Vilaseca, A. (2000). Hacia la competencia intercultural en el aprendizaje de una lengua extranjera. Estudio del choque cultural y los malentendidos. Madrid: Edinumen.

Ortí Teruel, R. (2004). "Estudio de la competencia intercultural a partir del análisis sociocultural de interacciones orales con arabohablantes", en Tonos, Revista Electrónica de Estudios Filológicos, 8: 1-16.

Pérez Ruiz, J. (2011). "Actitudes sociolingüísticas de la globalización en estudiantes taiwaneses de lenguas extranjeras. Resultados preliminares", en Tamkang Studies of Foreign Languages and Literatures, 17: 56-79.

Pérez Ruiz, J. (2012). El papel del oyente en la conversación china y española. Un estudio contrastivo sociolingüístico. Taipéi: Central book.

Pérez Ruiz, J. (2014). Corpus Oral Bilingüe Chino Español: una herramienta sociolingüística para investigaciones contrastivas. Taipéi: Central book.

Pérez Ruiz, J. (2014 en prensa). "La contribución del oyente a la interacción oral en español como lengua extranjera. Un estudio comparativo", en Revista Signos, 47,86: 1-28.

Rico Martín, A. M. (2005). "De la competencia intercultural en la adquisición de una segunda lengua o lengua extranjera: conceptos, metodología y revisión de métodos", en Porta Linguarum, 3: 79-94.

Ruiz San Emeterio, M. E. (2004). "Contenidos culturales en los métodos de ELE y en manuales de cultura y civilización española", en Red ELE, Revista electrónica de didáctica, 1: 1-10.

Sanhueza Henríquez, S. et al. (2012). "Dimensiones de la competencia comunicativa intercultural (CCI) y sus implicaciones para la práctica educativa", en Folios, 36, 2: 131-151.

Santamaría Martínez, Rocío (2008). La competencia sociocultural en el aula de español L2/LE: Una propuesta didáctica. Tesis doctoral inédita. Getafe: Universidad de Getafe.

Scollon, S. (1999). "Not to waste words or students: Confucian and Socratic discourse in the tertiary classroom", en E. Hinkel, (ed.): Culture in Second language Teaching and Learning. Cambridge: Cambridge University Press, 13-28. 
Seedhouse, P. (2004): The interactional architecture of the language classroom: A conversation analysis perspective. Malden, MA: Blackwell.

Sinclair, J. M. (2004). How to use corpora in Language Teaching. Amsterdam: John Benjamins Publishing.

Sinclair, J. y Coulthard, R. M. (1975). Towards an Analysis of discourse. The English used by teachers and pupils. Great Britain: Oxford University Press.

Stokoe, E. H. (2000). "Constructing Topicality in University Students' Small-group Discussion: A Conversation Analytic Approach", en Language and Education, 14, 3: 184-203.

Thompson, P. (2005). "Spoken Language Corpora", en M. Wynne (ed.): Developing Linguistic Corpora: a Guide to Good Practice. Oxford: Oxbow Books, 59-70.

Unamuno, V. (2003). Lengua, escuela y diversidad sociocultural. Hacia una educación lingüística critica. Barcelona: Graó.

Valls Campà, L. (2011). "Enseñanza/aprendizaje de la competencia comunicativa intercultural y análisis de actitudes", en MarcoELE Revista de Didáctica ELE, 13: 1-18. 